\title{
A Formal Modeling Framework for Anaerobic Degestion Systems
}

\author{
Khadidja Chaib Draa \\ Automatic Control Research Group \\ University of Luxembourg, luxembourg \\ khadidja.chaibdraa@uni.lu
}

\author{
Holger Voos \\ Automatic Control Research Group \\ University of Luxembourg, luxembourg \\ holger.voos@uni.lu
}

\begin{abstract}
The complexity of modeling the anaerobic digestion process meets the difficulty to describe and analyze them mathematically. In this paper, a simplified mathematical model for anaerobic digestion process of organic matter, in a continuous stirred tank reactor is proposed. With the aim of upgrading the produced biogas and integrate biogas plants in a virtual power plant, new control inputs reflecting addition of stimulating substrates (acetate and bicarbonate) are added. Based on two step (acedogenesis-methanogenesis) mass balance non linear model, a step-by-step model parameter's identification procedure is presented in the first step, then in the second step, the yield coefficients and the microbial growth rates are estimated when the later is assumed to be unknown a priori.
\end{abstract}

Keywords-Anaerobic Digestion; Non-linear Models; NonLinear Identification; Virtual Power Plants

\section{INTRODUCTION}

Anaerobic digestion process (AD) is a promising method for solving both energy and ecological problems. It consists on degrading organic matter (proteins, fats and carbohydrates) through a series of chemical and physicochemical reactions mediated, by anaerobic bacteria, to become it in biogas. The later is a potential energy which could be used to stabilize the power network. Despite the numerous advantages of the $\mathrm{AD}$ process, it is still bad responded in industry due to its instability characteristic, high sensor costs and difficult measurement procedures. Therefore, a mathematical modeling and software sensors are a great alternative to improve the process.

Modeling the AD process has been widely investigated in the last decades, we can find more than 70 models in the literature [1], and [2]. However, most of the existing models focus on the comprehension of the biological process, as is the case of $A D M 1$ for instance [3], and result in complex models unsuitable for control. Similarly much of those built for control purpose, allow it only by acting on the waste feed rate to the digester [5], [10]. This means that the constraints on the waste storage and the plant infrastructure were not considered, whereas this is far away for being true in the real life. However, some of waste treatment plants are constrained to treat a precise quantity of waste per day.

Therefore, to overcome the mentioned constraint, we propose a simplified model allowing the transfer of the experimental results from a laboratory scale to a real life operation scale. This is done, by the addition of two new control inputs reflecting the addition of a stimulating substrates in order to upgrade the produced biogas, while optimizing the system.

The first suggested control input is the acetate addition which is commonly used to increase the biogas quantity. However, the acetate addition causes $p H$ breakdown especially in low buffering capacity reactors, hence it is always accompanied by $p H$ increase (increase till $p H=8.5$ [9]) of the inlet waste. Often, the way $p H$ is increased is not optimal for the process since it does not take into account dynamics of inorganic carbon and alkalinity inside the reactor. Therefore, we added the second control input to control the bicarbonate addition in function of the reactor state.

The paper is organized as follows. In the next section II, we describe the proposed six order mass balance non-linear model followed by our proposed step-by-step identification procedure that is discussed in Section III. In Section IV, we propose an adaptive estimation of the yield coefficients and the specific reaction rates when no a priori knowledge about the biomass growth rates is available. Finally, we conclude the paper in Section V.

\section{Model Description}

It is well known that $\mathrm{AD}$ is a delicate process involving several microbial species [2], [3], and [7], and usually the model complexity is directly linked to the considered involved bacteria. Therefore, a two-step process (acedogenesis and methanogenesis) has often been reported in the literature to be suitable for the control purpose [5],[6], [7], and [10]. Hence, similarly the proposed model consider the following limiting steps:

1) Acidogenesis with reaction rate $r_{1}=\mu_{1} X_{1}$

$$
k_{1} S_{1} \stackrel{r_{7}}{\rightarrow} X_{1}+k_{2} S_{2}+k_{4} C O_{2}
$$

2) Methanogenesis with reaction rate $r_{2}=\mu_{2} X_{2}$

$$
k_{3} \mathrm{~S}_{2} \stackrel{r_{2}}{\rightarrow} \mathrm{X}_{2}+k_{5} \mathrm{CO}_{2}+k_{6} \mathrm{CH}_{4}
$$

where in the first step, acedogenic bacteria $\left(X_{1}\right)$ consume the organic substrate $\left(S_{1}\right)$ and produce volatile fatty acids (VFA) and $\mathrm{CO}_{2}$. In the second step, the produced VFA $\left(S_{2}\right)$ are consumed by methanogenic bacteria $\left(X_{2}\right)$ for growth and produce $\mathrm{CO}_{2}$ and methane. 
To model the considered limiting steps (1) and (2) while including the new control inputs, we assume that the reactor is perfectly stirred (thus biomass is uniformly distributed in the reactor), $p H$ and temperature $(T)$ range between 6 to 8 and 35 to $38^{\circ} \mathrm{C}$, respectively, and finally VFA $\left(S_{2}\right)$ behave like pure acetate.

\section{A. Model Equations}

We denote the state vector by $\xi=$ $\left[X_{1}, S_{1}, X_{2}, S_{2}, C, Z\right]^{T}$, where ${ }^{T}$ represents the transposed operator. The state vector is described by the following equations.

$$
\begin{aligned}
\dot{X}_{1}= & \left(\mu_{1}(\xi)-D\right) X_{1} \\
\dot{S}_{1}= & -k_{1} \mu_{1}(\xi) X_{1}-D S_{1}+D_{1} S_{1 i n} \\
\dot{X}_{2}= & \left(\mu_{2}(\xi)-D\right) X_{2} \\
\dot{S}_{2}= & k_{2} \mu_{1}(\xi) X_{1}-k_{3} \mu_{2}(\xi) X_{2}-D S_{2} \\
& +D_{1} S_{2 i n}+D_{2} S_{0}^{\prime} \\
\dot{C}= & k_{4} \mu_{1}(\xi) X_{1}+k_{5} \mu_{2}(\xi) X_{2}-D C \\
& +D_{1} C_{i n}+D_{3} C_{0}^{\prime}-Q_{c} \\
\dot{Z}= & -D Z+D_{1} Z_{i n}
\end{aligned}
$$

each one of the elements of Eq. (3) reflects the mass balance of its corresponding state. Here, $X_{i}\left(g . L^{-1}\right), S_{1}\left(g . L^{-1}\right), S_{2}$ (mmole. $\left.L^{-1}\right)$, and $\mu_{i}(\xi)\left(d a y^{-1}\right)$ are the bacteria concentration, the associated substrate concentrations, and the bacteria growth rates. Both rates are given by:

$$
\begin{cases}\mu_{1}\left(S_{1}\right)=\mu_{1 \max } \frac{S_{1}}{S_{1}+k_{s 1}} & \text { (Monod) } \\ \mu_{2}\left(S_{2}\right)=\mu_{2 \max } \frac{S_{2}}{S_{2}+k_{s 2}+\frac{S_{2}^{2}}{k_{I 2}}} & \text { (Haldane })\end{cases}
$$

With the undertaken $p H$ and the temperature $(T)$ assumptions, the total dissolved inorganic carbon concentration $C$ (mmole. $\left.L^{-1}\right)$ is the sum of bicarbonate (Bic) and the dissolved $\mathrm{CO}_{2}$ concentrations. Similarly, the alkalinity concentration $Z$ (mmole. $L^{-1}$ ) is the sum of bicarbonate (Bic) and dissociated acids $S_{2}$ (mmole. $\left.L^{-1}\right)$ mainly acetate. They are given by:

$$
\left\{\begin{array}{l}
Z=B i c+S_{2} \\
C=C O_{2}+B i c \\
p_{H}(\xi)=-\log _{10}\left(K_{b} \frac{C O_{2}}{B i c}\right)
\end{array}\right.
$$

The gaseous flow rates $Q_{c}$ and $Q_{M}$ of $\mathrm{CO}_{2}$ and $\mathrm{CH}_{4}$, respectively, are given as proposed in the $A M 2 G$ model [4].

$$
\left\{\begin{array}{l}
Q_{c}(\xi)=\frac{R T \gamma C O_{2}}{P_{T}+R T \gamma\left(K_{H} P_{T}-C O_{2}\right)} Q_{M} \\
Q_{M}(\xi)=k_{6} \mu_{2}(\xi) X_{2}
\end{array}\right.
$$

where $\gamma$ is the dimensionless parameter introduced by Hess [4] in order to reduce the expression complexity between the dissolved and the gaseous $\mathrm{CO}_{2}$.

$K_{i}$ are the yield coefficients defined in (I) and (II) with other parameters. $\xi_{\text {in }}$ are inlet waste concentrations fed to the digester with dilution rate $D_{1}, D_{2}$ is the dilution rate for the first added stimulate substrate (acetate) with concentration $S_{0}^{\prime}$, and $D_{3}$ is the dilution rate for the second added stimulate substrate (sodium bicarbonate) with concentration $C_{0}^{\prime} . D$ is the total dilution rate $\left(D=D_{1}+D_{2}+D_{3}\right)$.

\section{B. Model Steady States}

It is well known that biological processes exhibit multiple equilibrium states and the $\mathrm{AD}$ process takes part too. If we do not consider the washout of bacteria steady state $\left(X_{1}=0\right.$ or $\left.X_{2}=0\right)$, the steady states of the state model of the Eq. (3) are given by:

$$
\left\{\begin{array}{l}
\mu_{1}\left(S_{1}\right)=D \\
\mu_{2}\left(S_{2}\right)=D \\
S_{1}^{*}=k_{s 1} \frac{D}{\mu_{1 m a x}-D} \\
\frac{S_{2}^{* 2}}{k_{I 2}}+S^{*}\left(1-\frac{\mu_{2 m a x}}{D}\right)+k_{s 2}=0 \\
X_{1}^{*}=\frac{1}{k_{1}}\left(\frac{D_{1}}{D} S_{1 i n}-S_{1}^{*}\right) \\
X_{2}^{*}=\frac{1}{k_{3}}\left(\left(\frac{D_{1}}{D} S_{2 i n}-S_{2}^{*}\right)+\frac{k_{2}}{k_{1}}\left(\frac{D_{1}}{D} S_{1 i n}-S_{1}^{*}\right)\right) \\
\quad+\frac{D_{2}}{k_{3} D} S_{0}^{\prime} \\
Z^{*}=\frac{D_{1}}{D} Z_{i n} \\
C^{*}=\left(\frac{k_{4}}{k_{1}}+\frac{k_{5} k_{2}}{k_{3} k_{1}}\right)\left(\frac { D _ { 1 } } { k _ { 1 } } \left(\left(\frac{D_{1}}{D} S_{1 i n}-S_{1}^{*}\right)\right.\right. \\
\quad+\frac{k_{5}}{k_{3}}\left(\frac{D_{1}}{D} S_{2 i n}-S_{2}^{*}\right) \\
\quad+\frac{1}{D}\left(\frac{k_{5}}{k_{3}} S_{0}^{\prime}+D_{2}\right. \\
Q_{M}\left(\xi^{*}\right)=\frac{k_{6}}{k_{3}}\left(\left(D_{1} S_{2 i n}+S_{1}^{*}\right)\right)
\end{array}\right.
$$

Solutions of Eq. (7d) are the possible steady states for acetate $\left(S_{2}\right)$, where the largest one corresponds to methanogenesis inhibition phase (a detailed study of the corresponding equilibrium can be found in [4]).

Equilibrium of the dissolved inorganic carbon $(C)$ are obtained from Eqs (5), (6) and (7h). They are solutions of the following second degree equation:

$$
\begin{aligned}
0= & D \beta C^{* 2} \\
& -D C^{*}\left(P_{T}+\beta\left(K_{H} P_{T}+Z^{*}-S_{2}^{*}+k_{4} X_{1}^{*}\right)\right. \\
& -D C^{*}\left(+k_{5} X_{2}^{*}+k_{6} X_{2}^{*}+\frac{D_{1}}{D} C_{i n}+\frac{D_{3}}{D} C_{0}^{\prime}\right) \\
& +D\left(P_{T}+\beta\left(K_{H} P_{T}+Z^{*}-S_{2}^{*}\right)\left(k_{4} X_{1}^{*}+k_{5} X_{2}^{*}\right)\right. \\
& \left.+D\left(k_{6} X_{2}^{*}+\frac{D_{1}}{D} C_{i n}+\frac{D_{3}}{D} C_{0}^{\prime}\right)\right) \\
& +D \beta\left(Z^{*}-S_{2}^{*}\right) k_{6} X_{2}^{*}
\end{aligned}
$$


with $\beta=R T \gamma$.

In order to analyze (8), we rewrite it in the standard form as:

$$
0=f\left(C^{*}\right)=a C^{* 2}-b C^{*}+d
$$

where $a, b$ and $d$ are positive values.

By definition sum and product of this polynomial roots are positive, given by:

$$
C_{i}^{*}=\frac{b+(-1)^{i} \sqrt{\Delta}}{2 a}
$$

and evidently have positive real part.

To show that the roots (10) are real, we study the real function $f$.

It can be easily shown from (8) that $f(0)>0$ and $\lim _{C \rightarrow+\infty} f(C)=+\infty$. Moreover, the retained root should verify the positivity of $\mathrm{CO}_{2}$ flow rate $\left(Q_{c}^{*}>0\right)$ at the steady state. From (6), this condition is verified if:

$$
C^{*}<\frac{P_{T}+\beta\left(K_{H} P_{T}+Z^{*}-S_{2}^{*}\right)}{\beta}=C_{c}
$$

If, we calculate the polynomial (8) value for $C_{c}$, we find:

$$
f\left(C_{c}\right)=-\left(P_{T}+\beta K_{H} P_{T}\right) k_{6} X_{2}^{*}<0
$$

From (10), we know that the polynomial has two roots and it is of sign $-a$ in between. Hence, we deduce from (11) that $C_{1}^{*}<C_{c}<C_{2}^{*}$ and we conclude that $C_{1}$ is the only physically admissible root in the case of normal operating point.

\section{PARAMETERS IDENTIFICATION}

Since new inputs have been introduced in the model given by Eq. (3), it is mandatory to precise some of input's coefficients. Unfortunately, the not differentiability of parameters for such models is a well known issue. It has been reported in [13] [12] that different sets of coefficients can provide similar simulation results, and more, different coefficient values result for different particular organic matter [10], [11], [12]. Therefore a step-by-step identification procedure is often applied to solve this problem.

Hence, taking advantageous from the cascade structure of the model proposed in (3), we separate the parameters in different groups in order to identify them as independently as possible from each other. In the first step, we proceed as reported in [6] while estimating the parameters in steady state to force the model to correctly predict the equilibrium state to be reached by the system. In the second step, a calibration procedure based on non-linear optimization is to be performed.

While building the model, one should exploit as much as possible the available measurements and has to choose carefully the experiment conditions so as to cover as much as possible the range of the expecting operation situations.

Therefore, for the first step mean values of different steady states for $\overline{S_{1}}, \overline{S_{2}}, \bar{Z}, \bar{C}, \overline{p H}, \overline{Q_{c}}$ and $\overline{Q_{M}}$ are to be used.
The two separate considered groups of parameters are the kinetic parameters $\left(k_{s 1}, k_{s 2}, k_{I 2}, \mu_{1 \max }, \mu_{2 \max }\right)$ and the coefficient $\gamma$ in the first group, while the yield coefficients in the second one.

\section{A. Kinetic Parameters and $\gamma$ Identification}

From Eq. (7a) we obtain:

$$
\frac{1}{D}=\frac{1}{\mu_{1 \max }}+\frac{k_{s 1}}{\mu_{1 \max }} \frac{1}{\overline{S_{1}}}
$$

that can be used to estimate $\mu_{1 \max }$ and $k_{s 1}$ by a linear regression.

Similarly from Eq. (7b), we obtain the following relationship:

$$
\frac{1}{D}=\frac{1}{\mu_{2 \max }}+\frac{k_{s 2}}{\mu_{2 \max }} \frac{1}{\overline{S_{2}}}+\frac{1}{k_{I 2}} \frac{1}{\mu_{2 \max }} \overline{S_{2}}
$$

which is used to estimate $k_{I 2}, k_{s 2}$ and $\mu_{2 \max }$ by a linear regression too.

From Eqs. (5) and (6), we express the following relationship:

$$
\begin{aligned}
\frac{1}{\overline{Q_{c}}}= & \frac{1}{\gamma} \frac{P_{T}}{R T\left(\bar{C}-\bar{Z}+\overline{S_{2}}\right)} \frac{1}{\overline{Q_{M}}} \\
& +\left(\frac{K_{H} P_{T}-\bar{C}-\bar{Z}+\overline{S_{2}}}{\bar{C}-\bar{Z}+\overline{S_{2}}}\right) \frac{1}{\overline{Q_{M}}}
\end{aligned}
$$

from which a linear regression gives the value of the dimensionless parameter $\gamma$.

\section{B. Yield Coefficients Identification}

Using steady state equations given in Eqs. (6), (7h), and (7i), and the mean measurement values used previously, we can estimate the ratio of yield coefficients $\frac{k_{6}}{k_{3}}, \frac{k_{2}}{k_{1}}, \frac{k_{4}}{k_{1}}$ and $\frac{k_{5}}{k_{3}}$ by a linear regression applied to the following equations:

$$
\begin{aligned}
\overline{Q_{M}}= & \frac{k_{6}}{k_{3}}\left(\left(D_{1} S_{2 i n}-D \overline{S_{2}}\right)\right. \\
& \left.+\frac{k_{2}}{k_{1}}\left(D_{1} S_{1 i n}-D \overline{S_{1}}\right)\right)+\frac{k_{6}}{k_{3}} D_{2} S_{0}^{\prime} \\
\overline{Q_{c}}= & \left(\frac{k_{4}}{k_{1}}+\frac{k_{5} k_{2}}{k_{3} k_{1}}\right)\left(D_{1} S_{1 i n}-D \overline{S_{1}}\right) \\
+ & \frac{k_{5}}{k_{3}}\left(D_{1} S_{2 i n}-D \overline{S_{2}}+\left(\frac{k_{5}}{k_{3}} S_{0}^{\prime}+D_{3} C_{0}^{\prime}\right)\right. \\
+ & \left(D_{1} C_{i n}-D \bar{C}\right)
\end{aligned}
$$

So, the Eq. (16) can be rewritten using the methane flow rate measurement as follow:

$$
\overline{Q_{c}}=\frac{k_{2}}{k_{1}}\left(D_{1} S_{1} i n-D \overline{S_{1}}\right)+\frac{k_{5}}{k_{6}} \overline{Q_{M}}+\left(D_{1} C_{i n}-D \bar{C}\right)
$$

As it can be seen from Eqs. (15), (16) and (17), only the ratio of yield coefficients can be estimated from the used measurements. This issue was widely discussed in literature (see [6] and [10]). In fact, yield coefficients can be estimated only if acidogenic and methanogenic bacteria measurements $\left(\overline{X_{1}}\right.$ and $\overline{X_{2}}$ respectively) are available. 
Hence, we need to estimate the separate yield coefficients if we want to obtain the biomass concentration in the reactor. However, measuring biomass concentrations is unsuitable due to its complexity. Bernard et al. [6] proposed to use the volatile suspended solids (VSS) measurement as a rough approximation for the total biomass concentration $X_{1}+X_{2}$. Then, estimate each concentration separately using the ratio $v=0.2$ of acidogenic and methanogenic bacteria taken from [14]. Hence, by using the equilibrium steady state (7e), we have:

$$
v=\frac{\overline{X_{1}}}{\overline{X_{1}}+\overline{X_{2}}} \approx \frac{1}{k 1} \frac{\frac{D_{1}}{D} S_{1 i n}-S_{1}}{\overline{V S S}}
$$

if $\mathrm{v}$ is assumed to be constant $k_{1}$ is estimates by:

$$
k_{1}=\frac{1}{v} \frac{\frac{d_{1}}{d} s_{1 i n}-s_{1}}{\overline{v s s}}
$$

Now, using Eqs. (7e) and (7f), we get:

$$
\begin{aligned}
& v=\frac{k_{3}}{k_{1}} \times \\
& \frac{\frac{D_{1}}{D} S_{1 i n}-S_{1}}{\left(\frac{k_{3}}{k_{1}}+\frac{k_{2}}{k_{1}}\right)\left(\frac{D_{1}}{D} S_{1 i n}-S_{1}\right)+\frac{D_{2}}{D} S_{2 i n}-S_{2}+\frac{D_{2}}{D} S_{0}^{\prime}}
\end{aligned}
$$

thus $k_{3}$, can be estimated as follows:

$$
k_{3}=k_{1}\left(\frac{v}{1-v}\right)\left(\frac{k_{2}}{k_{1}}+\frac{\frac{D_{2}}{D} S_{2 i n}-S_{2}+\frac{D_{2}}{D} S_{0}^{\prime}}{\frac{D_{1}}{D} S_{1 i n}-S_{1}}\right)
$$

Finally, by using the yield coefficient ratios identified before, we can deduce all the yield coefficients estimates.

\section{Calibration}

Till now the yield coefficients for the steady state were estimated. This was possible by taking some assumptions like the constancy of the acidogenic and methanogenic ratio $v$, and the correlation between $V S S$ measurement and the biomass concentration in the reactor and, last but not least, the certainty of measurements at steady state. Therefore, to enable the model to predict as correct as possible the process behavior in both steady and transient states, we propose to calibrate the yield coefficients using online data measurements.

Usually, very restrictive information about the process can be obtained and only acetate $S_{2}$, alkalinity $Z$, and gaseous flow rates are measured for known inputs (often uncertain input concentrations). Therefore, we assume that $S_{2}, Z$ $Q_{c}$ and $Q_{M}$ are measured online to perform the nonlinear identification depicted in Fig. 1. where the criteriom $J$ to minimize is:

$$
\begin{aligned}
J=\min _{P} & \sum\left[L_{1}\left(S_{2 m e s}-S_{2 m d l}\right)^{2}\right. \\
& +L_{2}\left(Q_{M m e s}-Q_{M m d l}\right)^{2} \\
& +L_{3}\left(Q_{C m e s}-Q_{C m d l}\right)^{2} \\
& \left.+L_{4}\left(Z_{m e s}-Z_{m d l}\right)^{2}\right]
\end{aligned}
$$

with $L_{i}$ are weight coefficients, and $P$ a vector containing the parameters to be estimated.
Many optimization tools for non-linear identification with generated data from known models can be used to find the desired parameters. At this step, we use the previously found yield coefficients at steady state to initialize the non linear estimation algorithm and hence, we avoid biased estimates which usually occur when the algorithm is bad initialized. Actually, a simple iterative parameter estimation based on sensitivity analysis and non linear optimization methods has been proposed in [13]. We, suggest to use while initializing it by the previously found yield coefficients.

\section{AdAptive Parameters Estimation}

In this section, we describe briefly how to estimate simultaneously the yield coefficients and the growth rates when no a priori knowledge of the later analytic expression is available. This means that the operator have no knowledge about the factors which can influence the biomass growth. For seek of brevity, we describe the steps of the method without going in the mathematical details. First, lets rewrite the state model (3) in a matricial form:

$$
\begin{aligned}
{\left[\begin{array}{l}
\dot{X}_{1} \\
\dot{S}_{1} \\
\dot{X}_{2} \\
\dot{S}_{2} \\
\dot{C} \\
\dot{Z}
\end{array}\right]=} & {\left[\begin{array}{ll}
1 & 0 \\
-k_{1} & 0 \\
0 & 1 \\
k_{2} & -k_{3} \\
k_{4} & k_{5} \\
0 & 0
\end{array}\right]\left[\begin{array}{l}
\mu_{1} X_{1} \\
\mu_{2} X_{2}
\end{array}\right]-D\left[\begin{array}{l}
X_{1} \\
S_{1} \\
X_{2} \\
S_{2} \\
C \\
Z
\end{array}\right] } \\
& {\left[\begin{array}{l}
0 \\
D_{1} S_{1 i n} \\
0 \\
D_{1} S_{2 i n}+D_{2} S_{0}^{\prime} \\
D_{1} C_{1 i n}+D_{3} C_{0}^{\prime} \\
D_{1} Z_{\text {in }}
\end{array}\right]-\left[\begin{array}{l}
0 \\
0 \\
0 \\
0 \\
Q_{c} \\
0
\end{array}\right] }
\end{aligned}
$$

The general form of (23) is as follows:

$$
\xi=K \phi(\xi)-D \xi+F-Q
$$

Now, we split the state vector to tow sub-state vectors: $\xi_{1}$ contains the measurable states, and $\xi_{2}$ contains the remained not measurable states:

$$
\begin{aligned}
& \xi_{1}=K_{1} \phi(\xi)-D \xi_{1}+F_{1}-Q_{1} \\
& \xi_{2}=K_{2} \phi(\xi)-D \xi_{2}+F_{2}-Q_{2}
\end{aligned}
$$

Then, we introduce an auxiliary state $\zeta=A_{0} \xi_{a}+\xi_{b}$, where $\xi_{a}$ and $\xi_{b}$ are a nice partition allowing suppression of the unknown parameters from the dynamic of $\zeta$, hence $A_{0}=$ $-K_{2} K_{1}^{-1}$. Applying this model partition, widely discussed in [10], to the model (23) for $\xi_{a}=\left[X_{1}, X_{2}\right]^{T}$ and $\xi_{b}=$ $\left[S_{1}, S_{2}, C, Z\right]^{T}$, we get the following relationship:

$$
\zeta=\left[\begin{array}{ll}
k_{1} & 0 \\
-k_{2} & k_{3} \\
-k_{4} & -k_{5} \\
0 & 0
\end{array}\right]\left[\begin{array}{l}
X_{1} \\
X_{2}
\end{array}\right]+\left[\begin{array}{l}
S_{1} \\
S_{2} \\
C \\
Z
\end{array}\right]
$$




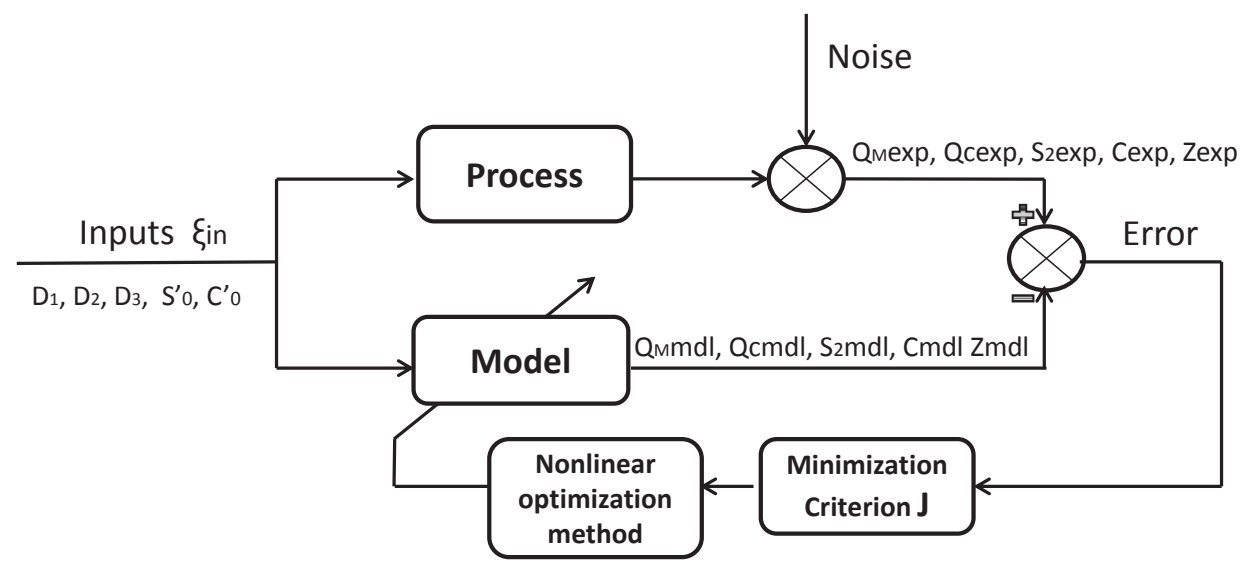

Figure 1. Non-Linear Identification Scheme.

and thus, the dynamic of $\zeta$ is given by:

$$
\begin{aligned}
{\left[\begin{array}{l}
\dot{\zeta}_{1} \\
\dot{\zeta}_{2} \\
\dot{\zeta}_{3} \\
\dot{\zeta}_{4}
\end{array}\right]=-D\left[\begin{array}{l}
\zeta_{1} \\
\zeta_{2} \\
\zeta_{3} \\
\zeta_{4}
\end{array}\right] } \\
+\left[\begin{array}{l}
D_{1} S_{1 i n} \\
D_{1} S_{2 i n}+D_{2} S_{0}^{\prime} \\
D_{1} C_{1 i n}+D_{3} C_{0}^{\prime}-Q_{c} \\
D_{1} Z_{\text {in }}
\end{array}\right]
\end{aligned}
$$

Actually, now we can proceed for the yield and growth rates estimations using the new system containing the auxiliary state $\zeta$ and the measured state $\xi_{1}$ as described below. But, before proceeding with that we rewrite the product $\mu_{i} X_{i}$ as the product of the specific reaction $\alpha_{i}$ and the responsible components involved in the chemical reaction [10], thus we have:

$$
\begin{aligned}
& \mu_{1} X_{1}=\alpha_{1} S_{1} X_{1} \\
& \mu_{2} X_{2}=\alpha_{2} S_{2} X_{2}
\end{aligned}
$$

It has been already proved in the previous sections that yield coefficients can be estimated only if the biomass concentrations are measured. Hence, we assumed that $\xi_{1}=$ $\left[X_{1}, X_{2}, C\right]^{T}$ where using (28) its dynamic is given by:

$$
\begin{aligned}
{\left[\begin{array}{c}
\dot{X}_{1} \\
\dot{X}_{2} \\
\dot{C}
\end{array}\right]=} & {\left[\begin{array}{ll}
1 & 0 \\
0 & 1 \\
k_{4} & k_{5}
\end{array}\right]\left[\begin{array}{l}
\alpha_{1} S_{1} X_{1} \\
\alpha_{2} S_{2} X_{2}
\end{array}\right]-D\left[\begin{array}{l}
X_{1} \\
X_{2} \\
C
\end{array}\right] } \\
+ & {\left[\begin{array}{l}
0 \\
0 \\
D_{1} C_{1 i n}+D_{3} C_{0}^{\prime}
\end{array}\right]-\left[\begin{array}{l}
0 \\
0 \\
Q_{c}
\end{array}\right] }
\end{aligned}
$$

Hence, the matrices $A_{1}$ and $A_{2}$ are the following:

$$
A_{1}=\left[\begin{array}{lll}
k_{1} & 0 & 0 \\
-k_{2} & -k_{3} & 0 \\
-k_{2} & k_{5} & 1 \\
0 & 0 & 0
\end{array}\right], A_{2}=\left[\begin{array}{lll}
1 & 0 & 0 \\
0 & 1 & 0 \\
0 & 0 & 0 \\
1 & 0 & 0
\end{array}\right]
$$

Now the new state model to be processed contains $\zeta$ and $\xi_{1}$ described by (26) and (29), respectively.

In order to estimate the unknown yield coefficients and the specific reaction rates, we separate the known measurements from the unknown parameters. Therefore, the missed measurements are expressed in function of $\xi_{1}$ and $\zeta$. Hence, the dynamic expression of $\xi_{1}$ is given as follows:

$$
\xi_{1}=\Psi\left(\xi_{1}, \zeta\right) \theta-D \xi_{1}+F_{1}-Q_{1}
$$

with $\Psi\left(\xi_{1}, \zeta\right)$ is given by (32), $\theta=\left[\alpha_{1}, k_{1} \alpha_{1}, \alpha_{2}, k_{2} \alpha_{2}\right.$, $\left.k_{3} \alpha_{2}, k_{4} \alpha_{1}, k_{1} k_{4} \alpha_{1}, k_{5} \alpha_{2}, k_{2} k_{5} \alpha_{2}, k_{3} k_{5} \alpha_{2}\right]^{T}, F_{1}=[0,0$, $\left.D_{1} C_{i n}+D_{3}+C_{0}^{\prime}\right]^{T}$ and finally $Q_{1}=\left[0,0, Q_{c}\right]^{T}$.

On the grounds of the previous transformations and definitions the vector $\theta$ (hence the unknown parameters) can be estimated using an observer based estimator like the following:

$$
\begin{aligned}
& \dot{\hat{\zeta}}=-D \hat{\zeta}+F_{b}-Q_{b} \\
& \hat{\xi}_{1}=\Psi\left(\xi_{1}, \hat{\zeta}\right) \hat{\theta}-D \xi_{1}+F_{1}-Q_{1}-\Omega\left(\xi_{1}-\hat{\xi}_{1}\right) \\
& \dot{\hat{\theta}}=\Psi\left(\xi_{1}, \hat{\zeta}\right) \Gamma\left(\xi_{1}-\hat{\xi}_{1}\right)
\end{aligned}
$$

where $\Omega$ and $\Gamma$ are design parameters, to be fixed by the designer so that the states given by the estimated parameters fit well the experiments data.

\section{CONClusion}

In order to facilitate the modeling aspect to control efficiently the anaerobic digestion process, we proposed a mathematical (formal) framework that describes how new stimulating substrates (acetate and bicarbonate) can be added to this process. This was motivated by the aim of controlling the quantity and the quality of the produced biogas from treatment plants in order to integrate them in a virtual power plant. To identify the model parameters, we proposed a step-by-step identification procedure based on two steps (acedogenesis-methanogenesis) mass balance 


$$
\phi=\left[\begin{array}{llllllllll}
X_{1} \zeta_{1} & X_{1}^{2} & 0 & 0 & 0 & 0 & 0 & 0 & 0 & 0 \\
0 & 0 & X_{2} \zeta_{2} & X_{1} X_{2} & -X_{2}^{2} & 0 & 0 & 0 & 0 & 0 \\
0 & 0 & 0 & 0 & 0 & X_{1} \zeta_{1} & -X_{1}^{2} & X_{2} \zeta_{2} & X_{1} X_{2} & -X_{2}^{2}
\end{array}\right]
$$

non linear model. Further, we described an online adaptive algorithm for yield coefficients and specific reaction rates estimation when no knowledge about the factors influencing the biomass behavior is available.

The research that we have initiated in this paper sets the fundamentals for further fully automatic modelling and analysis of biogas systems. First, we intend to validate our model on more realistic parameter. Then, we will propose new strategies of control to integrate the biogas plants in a virtual power plant to stabilize the power network.

\section{ACKNOWLEDGMENT}

The research leading to the results presented in this work received funding from $\mathrm{XXX}$.

\section{NOMENCLATURE}

\begin{tabular}{ll}
\hline \hline Acronyms & Definition \\
\hline$k_{1}$ & Yield for substrate degradation \\
$k_{2}$ & Yield for VFA production \\
$k_{3}$ & Yield for VFA consumption \\
$k_{4}$ & Yield for $C \mathrm{CO}_{2}$ production \\
$k_{5}$ & Yield for $C \mathrm{O}_{2}$ production \\
$k_{6}$ & Yield for $C h_{4}$ production \\
$\mu_{1 \max }$ & Maximum acedogenic bacteria growth rate \\
$\mu_{2 \max }$ & Maximum methanogenic bacteria growth rate \\
$k_{s 1}$ & Half saturation constant associated with $S_{1}$ \\
$k_{s 2}$ & Half saturation constant associated with $S_{2}$ \\
$K_{I 2}$ & Inhibition constant associated with $S_{2}$ \\
$K_{b}$ & Acidity constant of bicarbonate \\
$K_{H}$ & Henry s constant \\
$R$ & Gas constant \\
$P_{T}$ & Total preasure \\
$\mathrm{T}$ & Temperature \\
$\gamma$ & dimonsionless parameter introduced by Hess [4] \\
\hline \hline
\end{tabular}

TABLE I

NOMENCLATURE

\section{REFERENCES}

[1] F. Prause 1997. Mathematische Modellierung des anaeroben Reinigungsprozesses Darstellung bisheriger Ansätze und Anwendungen eines ausgewählten Modells am Beispiel der KA Marne. Diplomarbeit. Institut für Siedlungswasserwirtschaft und Abfalltechnik der Universität Hannover.

[2] S. Weinrich, M. Nelles, R.F. Jacobi, E. Mauky 2013. Praxisnahe Modellierung von Biogasanlagen Vergleich unterschiedlicher Modellanstze. Ifak Workshop in Leipzig.

[3] D.J. Batstone, J. Keller, I. Angelidaki, S.V. Kalyuzhnyi, S.G. Pavlostathis, A. Rozzi, W.T.M. Sanders, H. Siegrist, V.A. Vavilin 2002. Anaerobic Digestion Model No. 1. IWA Publishing, IWA STR No. 13.

[4] J. Hess, O. Bernard, J. P. Steyer 2008. Thse: Modlisation de la Qualit du Biogas Produit par un Fermenteur Mthanogne et sa Stratgie de Rgulation en Vue de sa Valorisation. Universit de Nice - Sophia Antipolis.

\begin{tabular}{ll}
\hline \hline Acronyms & Units \\
\hline$k_{1}$ & g. $g^{-1}$ of acidogenic bacteria \\
$k_{2}$ & mmole. $g^{-1}$ of acidogenic bacteria \\
$k_{3}$ & mmole. $g^{-1}$ of methanogenic bacteria \\
$k_{4}$ & mmole. $g^{-1}$ \\
$k_{5}$ & mmole. $g^{-1}$ \\
$k_{6}$ & mmole. $g^{-1}$ \\
$\mu_{1 \max }$ & day ${ }^{-1}$ \\
$\mu_{2 \max }$ & day \\
$k_{s 1}$ & g.l \\
$k_{s 2}$ & mmol. $l^{-1}$ \\
$K_{I 2}$ & mmol.l ${ }^{-1}$ \\
$K_{H}$ & mmole. $L^{-1} \cdot$ atm ${ }^{-1}$ \\
$R$ & L.atm. $K^{-1} \cdot$ mol $^{\text {mol }}$ \\
$P_{T}$ & atm \\
$\mathrm{T}$ & Kelvin \\
\hline \hline
\end{tabular}

TABLE II

NOMENCLATURE-CONTINUATION

[5] P. Renard, V. Van Breusegem, M.T Nguyen, E.J Nyns 1991. Implementation of an adaptive Controller for the Startup and Steady-State Running of a Biomethanation Process Operated in the CSTR Mode. Biotechnology and Bioengineering, Vol. 38, P.805-812.

[6] O. Bernard, Z. Hadj-Sadok, D. Dochain, A. Genovesi and J. Steyer, 2001. Dynamical Model Development and Parameter Identification for an Anaerobic Wastewater Treatment Process. Biotechnology and Bioengineering, Vol. 75, No.4.

[7] S. Marsili-Libelli, S. Beni 1994. Shock load modelling in the anaerobic digestion process. Ecological Modelling 84 (1996) 215-232.

[8] J. Hess,O. Bernard 2006. Mechanistic kLa Modelling in an Up-flow Anaerobic Digester. Proceedings of the 5th Symposium on Mathematical Modeling, Vienna, Austria, MATHMOD.

[9] V. Lubenova, I. Simeonov, I. Queinnec 2002. Two Step Parameter and State Estimation of The Anaerobic Digestion. IFAC, $15^{\text {th }}$ Terminal World Congress, Barcelona, Spain.

[10] G. Bastin, D. Dochain 1991. On-line Estimation and Adaptive Control of Bioreactors. Elsevier Science Publishers, Amsterdam and New York.

[11] N. Noykova 2002. Modelling and Identification of Microbial Population Dynamics in Wastewaters Treatment. Thesis E9, University of Turku, Institute for Applied Mathematics, Finland.

[12] I. Simeonov, V. Lubenova, I. Queinnec 2009. Parameter and State Estimation of an Anaerobic Digestion of Organic Wastes Model with Addition of Stimulating Substances BIO Automation (2009) 12, 88-105.

[13] I. Simeonov 1999. Mathematical Modelling and Parameters Estimation of Anaerobic Fermentation Processes Bioprocess Engineering, 21, 377-381.

[14] J. Sanchez, S. Arijo, M. Munoz, M. Morinigo, J. Boirrego 1994. Microbial Colonization of Different Support Material Used to Enhance the Methanogenic Process. Appl Microbial Biotechnol, 41, 480-486. 